\title{
STANDARDISED QUESTIONNAIRE FOR THE EARLY IDENTIFICATION OF FEEDING PROBLEMS AND GASTROINTESTINAL SYMPTOMS IN CHILDREN WITH AUTISM AND THE IMPACT OF THESE PROBLEMS ON FAMILY LIFE
}

\author{
N.S. Mohamad Nor, A.S. Le Couteur \\ Institute of Health and Society, Newcastle University, Newcastle upon Tyne, UK
}

Background and aim: Managing feeding problems and gastrointestinal (GI) symptoms in children with Autism Spectrum Disorders (ASD) is a challenging process for professionals and parents. These problems also have psychosocial impact on the family life. Our aim is to develop a new standardised questionnaire called "The brief structured questionnaire for the early identification of feeding problems and GI symptoms in primary school children with ASD (BEFG-ASD)" that we hope will assist professionals in a systematic way.

Methods: The process for the development of the BEFG-ASD involved several stages including a comprehensive literature review. Once an agreed draft BEFG-ASD had been developed, it was reviewed using a Delphi technique. Two panels of experts (professionals and parents of children with ASD) were asked to rate their opinion of the BEFG-ASD in two rounds using a standard review format.

Results: Overall, the parent and professional experts rated questions in the BEFG-ASD as 'important' or 'very important' (Mean score: 1.0-2.0). Experts also made suggestions to refine the wording of some items. The final revision of the BEFG-ASD consists of 50 items in fourteen (14) sub-domains of feeding problems, GI symptoms and the impact. This version was then pre-tested among professionals and parents $(\mathrm{n}=16)$.

Conclusion and next step: Our standardised questionnaire was been successfully developed. The content and face validity was established. The BEFG-ASD is currently being field-tested with professionals and parents in North East England to evaluate the psychometric properties. We hope that the BEFG-ASD will assist professionals in UK and further afield. 